\title{
Short-chain fatty acids produced in vitro from fibre residues obtained from mixed diets containing different breads and in human faeces during the ingestion of the diets
}

\author{
Elisabeth Wisker ${ }^{1 *}$, Martina Daniel ${ }^{1}$, Gerhard Rave ${ }^{2}$ and Walter Feldheim ${ }^{1}$ \\ Christian Albrechts-University of Kiel, ${ }^{1}$ Institute of Human Nutrition and Food Science, Düsternbrooker Weg 17 , \\ D-24105 Kiel, Germany, ${ }^{2}$ Variationsstatistik, Hermann-Rodewaldstraße 9, D-24098 Kiel, Germany \\ (Received 18 December 1998 - Revised 7 September 1999 - Accepted 8 December 1999)
}

\begin{abstract}
It was studied whether the type of bread (i.e. a low-fibre wheat-rye mixed bread and coarse or fine wholemeal rye bread) either as part of a diet or alone, had an influence on the short-chain fatty acids (SCFA) produced during in vitro fermentation. Fermentation substrates were dietary fibre residues obtained from diets and breads. In addition, it was investigated whether the faecal SCFA pattern in the inoculum donors, who ingested the experimental diets, could be predicted by in vitro fermentation. Yields of SCFA in vitro were $0.51-0.62 \mathrm{~g} / \mathrm{g}$ fermented polysaccharide. In vitro, the molar ratios of butyrate were higher for the two high-fibre diets containing coarse or fine wholemeal bread than for the low fibre diet containing wheat-rye mixed bread; the difference was significant for the coarse $(P<0.01)$, but not for the fine bread diet $(P=0.0678)$. The coarse wholemeal bread alone produced a higher molar ratio of butyrate than the fine wholemeal bread $(P<0.05)$ and the wheat-rye mixed bread $(P<0.01)$. Ingestion by the inoculum donors of the diets containing wholemeal bread led to higher faecal butyrate ratios (molar ratios: coarse bread diet 19.6, fine bread diet 17.7) compared with the wheat-rye mixed bread-containing diet (14.9), but the differences between the diets were not significant. For the diets investigated, there were no significant differences between faecal and in vitro SCFA patterns.
\end{abstract}

Fermentation: Short-chain fatty acids: Mixed diets: Faeces

Colonic fermentation of NSP and resistant starch (RS) results in the formation of variable amounts and proportions of the short-chain fatty acids (SCFA) acetate, propionate and butyrate. Butyrate is of particular interest because it is the preferred fuel of distal colonic cells (Roediger, 1982) and is supposed to be involved in the protective effect of fibre against colon cancer (Bingham, 1996). Because the influence of dietary carbohydrates on the SCFA production in the human colon is difficult to investigate, SCFA formation is often studied in vitro using human faecal inocula (e.g. Englyst et al. 1987; Adiotomre et al. 1990; McBurney \& Thompson, 1990). An alternative approach is the determination of faecal SCFA (e.g. Scheppach et al. 1988; Cummings et al. 1996; Heijnen et al. 1998). However, the ingestion of several types of unavailable polysaccharides led to faecal SCFA patterns that differed from those obtained in vitro (Cummings et al. 1996; Heijnen et al. 1998). This may have been due to the fact that most fermentation occurs in the proximal colon and that at least $95 \%$ of the SCFA produced are rapidly absorbed (Ruppin et al. 1980). Therefore, faecal SCFA do not reflect SCFA production in the proximal colon, but they may reflect the substrates available in the distal large intestine (McIntyre et al. 1991; Macfarlane et al. 1994).

Recently, we reported that the extent of degradation in human subjects of NSP in five mixed diets and in single fibre sources could be predicted by an in vitro batch system (Wisker et al. 1998). In one part of this study, in vitro fermentations were performed during the ingestion of the experimental diets by the inoculum donors. Coarse or fine wholemeal rye bread or a low fibre wheat-rye mixed bread were the predominant fibre sources in these diets. An additional aim of that study was to investigate whether the type of bread present in the diets had an influence on the SCFA produced from fermentation. Therefore, we determined the formation in vitro of SCFA from fibre residues obtained from the diets and the corresponding breads. We also measured faecal SCFA in the inoculum donors to see whether for these fibre sources faecal SCFA patterns could be predicted by the SCFA pattern obtained in vitro. In contrast to readily fermentable fibres, lignified fibres such as those present in wholemeal breads are slowly fermented (Van

\footnotetext{
Abbreviations: RS, resistant starch; SCFA, short-chain fatty acids.

* Corresponding author: Dr E. Wisker, fax +49 431880 1528, e-mail ewisker@nutrfoodsc.uni-kiel.de
} 
Soest et al. 1982), so it might be expected that at least parts of them would reach the distal colon and thus might have an effect on faecal SCFA.

\section{Experimental methods}

\section{Subjects and study plan}

Seven apparently healthy, free-living female students aged 22-31 years participated in the experiments. Subjects had not taken antibiotics for at least 6 weeks before the beginning of the experiments. The study was approved by the Ethics Committee of the Medical Faculty of the University of Kiel. Details of the studies have been reported previously (Wisker et al. 1998). In brief, the study included three experimental periods during which a low-fibre diet and two high-fibre diets were eaten. Subjects were randomly assigned to start on one of the experimental diets and then followed a different sequence of diets. Each study period lasted $22 \mathrm{~d}$; periods were separated from each other by $21 \mathrm{~d}$. In vitro fermentations were carried out between day 17 and day 21 of each experimental period. During this time, from each subject one faecal sample was obtained for the in vitro fermentations and for faecal SCFA analysis.

\section{Diets}

The low-fibre diet and the high-fibre diets differed in the amounts and types of bread. Wholemeal rye breads were baked from one single batch of rye milled to two different particle sizes (coarse bread: $50 \%$ of particles $>2 \mathrm{~mm}$, $90 \%>1 \mathrm{~mm}$; fine bread: $99 \%$ of particles $<0.5 \mathrm{~mm}$, $58 \%<0.2 \mathrm{~mm}$ ) (Federal Institute of Grain, Potato and Fat Research, Detmold, Germany). Wheat-rye mixed bread was typical German low-fibre bread. Bread provided 60, 82 and $83 \%$ of NSP consumed during the low-fibre diet and the high-fibre diets containing coarse or fine wholemeal rye bread respectively. The remainder of NSP was provided by vegetables, fruits and potatoes. Although during each dietary period all subjects consumed the same amount of fibrecontaining foods, there were minor differences between the subjects in the NSP intake. This occurred because the study was performed in a crossover design; the NSP content of fruits, vegetables and potatoes which were bought fresh varied somewhat between the three dietary periods. Intake of NSP was 16.7 (SD 0.8), 38.2 (SD 0.8) and 37.7 (SD 0.7) g/d (means $\pm \mathrm{SD}$ ) for the low-fibre and the high-fibre diets containing coarse or fine wholemeal rye bread respectively.

\section{Fermentation substrates}

Dietary fibre residues obtained from freeze-dried mixtures of the fibre-containing foods in the same proportions as consumed during the in vivo studies and from the breads were fermented in vitro. With the exception of the substrates prepared from coarse bread, the freeze-dried foods were milled through an $0.5 \mathrm{~mm}$ screen. For the diet containing coarse wholemeal bread, bread was dried separately from the other foods and crumbled by hand to a particle size resembling that of the coarse meal used for bread making. Bread and the other freeze-dried ground foods were mixed together before the preparation of the dietary fibre residues. Coarse bread alone was also comminuted to the particle size of the coarse meal. Total dietary fibre residues were prepared according to a gravimetric method for dietary fibre analysis (Prosky et al. 1985). For each diet and the fibre concentrate respectively, dietary fibre residues were prepared in an amount sufficient for all incubations and kept in a desiccator until used.

\section{Faecal inoculum}

Freshly passed faeces were immediately homogenized for 3 min with four times their weight of distilled water, filtered through four layers of cheesecloth to remove particles and immediately used as inoculum. Inoculum preparation was performed under a constant flow of $\mathrm{CO}_{2}$. Aliquots of the faecal homogenates were analysed for SCFA as described later.

\section{Fermentations}

In each experimental period, portions of each faecal homogenate obtained from the individual subjects were incubated with the dietary fibre residues prepared from the fibre sources eaten by these subjects during this period. Incubations were performed for 0,24 and $48 \mathrm{~h}$. Flasks without added substrate as blanks were also incubated for each time point with each inoculum source. In the present work, SCFA formation is reported for $24 \mathrm{~h}$ in vitro fermentations (see p. 35).

Fermentations were conducted in $125 \mathrm{ml}$ Erlenmeyer flasks using the technique and the solutions described by Goering \& Van Soest (1970). The basic components of this system include substrate, a carbohydrate-free culture medium, reducing solution and faecal inoculum as described previously (Wisker et al. 1998). Culture medium (32 ml) was added to $400 \mathrm{mg}$ substrate (dietary fibre residue) 12 to $24 \mathrm{~h}$ before the start of the incubation to ensure complete hydration of the samples. Flasks were sealed with parafilm and stored in the refrigerator to limit the possibility of microbial growth. At $1-2 \mathrm{~h}$ before inoculation, the bottles were placed in a $37^{\circ} \mathrm{C}$ shaking water bath, reducing solution $(1.6 \mathrm{ml})$ was added and then flasks were sealed with rubber stoppers. Stoppers were fitted with three openings (an inlet tube, a Bunsen valve, and a gassing tube) connected to a common manifold as described by Goering \& Van Soest (1970). The manifold was connected to supply of $\mathrm{CO}_{2}$, and flasks were bubbled with $\mathrm{CO}_{2}$. Into each flask $8 \mathrm{ml}$ faecal suspension were injected through the inlet tube, and incubations were performed under a steady stream of $\mathrm{CO}_{2}(3-4 \mathrm{ml} /$ $\mathrm{min}$ ). Flasks were gently shaken (shaking rate $50 / \mathrm{min}$ ). Fermentation was stopped by adding $1 \mathrm{ml}$ ethylmercurithiosalicylate $(10 \mathrm{~g} / \mathrm{l})$. From each incubation flask, a $1.0 \mathrm{ml}$ portion was removed for SCFA analysis. The remaining contents of the flasks were quantitatively transferred to pretared beakers, freeze-dried and used for the determination of residual NSP.

For the determination of faecal SCFA, $9 \mathrm{ml}$ faecal homogenate was mixed with $1 \mathrm{ml}$ ethylmercurithiosalicylate $(10 \mathrm{~g} / \mathrm{l})$ and $10 \mathrm{ml}$ distilled water and vortexed vigorously. Then the samples were centrifuged at 12000 r.p.m. for 
15 min and the supernatants used for SCFA analyses as described below.

\section{Chemical analyses}

Freeze-dried samples of food and faeces were milled through an $0.5 \mathrm{~mm}$ mesh screen. DM, N, neutral NSP, uronic acids and RS were assayed as described previously (Wisker et al. 1998). For the determination of SCFA, $0.5 \mathrm{ml}$ portions of the in vitro fermentation samples or supernatants of diluted faecal homogenates were mixed with $2.5 \mathrm{ml}$ oxalic acid $(0.03 \mathrm{~mol} / \mathrm{l})$ and $0.1 \mathrm{ml}$ internal standard $(30 \mu \mathrm{l}$ pivalic acid/ $\mathrm{ml})$ and centrifuged $(4000 \mathrm{~g})$ for $10 \mathrm{~min}$. Supernatants were used for the measurements of SCFA by GLC. Glass column packing material was $4 \%$ Carbowax 20M/80/120 Carbopack B-DA (Supelco, Bellefonte, PA, USA). For quantitation, analyses were performed with known SCFA standard solutions. All samples were analysed in duplicate.

\section{Calculations}

The production $\left(\mathrm{P}_{\mathrm{i}}\right)$ of SCFA in vitro was calculated as: $\mathrm{P}_{\mathrm{i}}=$ $\left(\mathrm{SCFA}_{24}-\mathrm{B}_{24}\right)-\left(\mathrm{SCFA}_{0}-\mathrm{B}_{0}\right)$, where $\mathrm{SCFA}_{0}$ and $\mathrm{SCFA}_{24}$ are the SCFA contents in flasks containing substrates at 0 and $24 \mathrm{~h}$ respectively, and $\mathrm{B}_{0}$ and $\mathrm{B}_{24}$ are SCFA contents in blank flasks without added substrate at 0 and $24 \mathrm{~h}$ respectively. Molar ratios of SCFA comprised acetate, propionate and butyrate. The differences between the molar ratios of SCFA in vitro and in faecal suspensions were calculated for the same subject, diet, and period.

\section{Statistical analyses}

The statistical analyses were performed using the procedure MIXED of SAS Release 6.12 (Statistical Analysis Systems Institute Inc., Cary, NC, USA). Least squares means were estimated and compared by $t$ test. The Bonferroni-Holm adjustment of $\mathrm{P}$ values was used for all multiple comparisons. Data were analysed as crossover design with subjects as random factor, and study periods and substrates as fixed factors. The comparison faecal $v$. in vitro SCFA pattern was done as paired comparison by analysing the differences between the molar ratio of SCFA in vitro and in faecal suspensions for the same subject, diet, and period. For these differences, least squares means were estimated and tested by $t$ test whether they were significantly different from zero. Differences were regarded as significant at $P<0.05$.

\section{Results}

The amounts of polysaccharides fermented in vitro are shown in Table 1 . Between 4.16 and $4.80 \mathrm{~g} / \mathrm{l}$ of polysaccharides were fermented. For the low-fibre diet and the corresponding wheat-rye mixed bread, less pentoses and more hexoses were broken down compared with the highfibre diets containing wholemeal breads and the corresponding breads. The quantities of degraded uronic acids were between 0.54 and $0.61 \mathrm{~g}$ for the diets, and were only very small for the breads. In all substrates, RS was completely degraded.

The amounts of acetate, propionate, and butyrate and the molar ratios of these SCFA produced in vitro are given in Table 2. SCFA concentrations ( $\mathrm{mmol} / \mathrm{l})$ and the quantities produced per $\mathrm{g}$ fermented polysaccharide (NSP $+\mathrm{RS}$ ) were significantly higher for the low-fibre diet than for the highfibre diets containing coarse $(P<0.01)$ or fine wholemeal bread $(P<0.05)$ respectively. Compared with the low-fibre diet, the molar ratios of butyrate were higher for the two high-fibre diets; the difference was significant for the coarse $(P<0.01)$, but not for the fine bread diet $(P=0.0678)$. The coarse bread diet yielded a non-significantly $(P=0.0678)$ higher molar ratio of butyrate than the fine bread diet.

For the single breads, no significant differences were found in the concentrations ( $\mathrm{mmol} / \mathrm{l})$ of total SCFA, but the amounts produced per $\mathrm{g}$ fermented polysaccharide were higher for the wheat-rye mixed bread than for the wholemeal breads. There were no differences between the breads in the molar ratios of acetate. The coarse wholemeal bread produced a higher percentage of butyrate and a lower percentage of propionate compared with the fine wholemeal

Table 1. Non-starch polysaccharides and resistant starch fermented in vitro* (Mean values with their standard errors for seven subjects)

\begin{tabular}{|c|c|c|c|c|c|c|c|c|c|c|}
\hline \multirow{3}{*}{ Substrates $(g / l) \ldots$} & \multicolumn{6}{|c|}{ Non-starch polysaccharides } & & & & \\
\hline & \multicolumn{2}{|c|}{ Pentoses† } & \multicolumn{2}{|c|}{ Hexoses $\ddagger$} & \multicolumn{2}{|c|}{ Uronic acids } & \multicolumn{2}{|c|}{ Resistant starch } & \multicolumn{2}{|c|}{$\begin{array}{c}\text { Total } \\
\text { polysaccharides }\end{array}$} \\
\hline & Mean & SD & Mean & SD & Mean & SD & Mean & SD & Mean & SD \\
\hline \multicolumn{11}{|l|}{ Diets } \\
\hline Low-fibre diet & 1.58 & 0.02 & 1.44 & 0.11 & 0.61 & 0.01 & 0.75 & 0.00 & 4.38 & 0.14 \\
\hline High-fibre diet (coarse wholemeal bread) & 2.03 & 0.06 & 1.20 & 0.17 & 0.54 & 0.01 & 1.00 & 0.00 & 4.77 & 0.24 \\
\hline High-fibre diet (fine wholemeal bread) & $2 \cdot 31$ & 0.03 & 1.25 & 0.13 & 0.54 & 0.01 & 0.51 & 0.00 & 4.61 & 0.13 \\
\hline \multicolumn{11}{|l|}{ Breads } \\
\hline Wheat-rye mixed bread & 2.01 & 0.02 & 1.52 & $0 \cdot 10$ & 0.07 & 0.03 & 0.55 & 0.00 & $4 \cdot 16$ & 0.13 \\
\hline Coarse wholemeal bread & 2.45 & 0.05 & 1.00 & $0 \cdot 13$ & 0.05 & 0.02 & 0.99 & 0.00 & 4.49 & 0.17 \\
\hline Fine wholemeal bread & $3 \cdot 15$ & 0.03 & 1.13 & $0 \cdot 11$ & 0.08 & 0.01 & 0.43 & 0.00 & 4.80 & 0.11 \\
\hline
\end{tabular}

* Data are calulated from the composition of substrates and their fermentability as presented by Wisker et al. 1998. For details of substrate preparation see p. 32 , and for details of diets see p. 32 and Wisker et al. 1998.

$\uparrow$ Sum of arabinose and xylose.

$\ddagger$ Sum of glucose, galactose and mannose. 
Table 2. Short-chain fatty acids (SCFA) produced during the fermentation in vitro of substrates prepared from the mixed diets and single fibre sources contained in these diets $\dagger$

(Least squares means with their standard errors for seven subjects)

\begin{tabular}{|c|c|c|c|c|c|}
\hline \multirow[t]{2}{*}{ Fermentation substrate ... } & \multicolumn{2}{|c|}{ Total SCFA } & \multirow[t]{2}{*}{ Acetate } & \multirow{2}{*}{$\begin{array}{l}\text { Propionate } \\
\text { molar ratio }\end{array}$} & \multirow[t]{2}{*}{ Butyrate } \\
\hline & $\mathrm{mmol} / \mathrm{l}$ & $\begin{array}{c}\mathrm{mmol} / \mathrm{g} \text { fermented } \\
\text { polysaccharide§ }\end{array}$ & & & \\
\hline \multicolumn{6}{|l|}{ Diets } \\
\hline Low-fibre diet & $46 \cdot 7^{\mathrm{a}}$ & $10 \cdot 4^{\mathrm{a}}$ & $69 \cdot 1$ & $19 \cdot 5$ & $11.5^{b}$ \\
\hline High-fibre diet (coarse wholemeal rye bread) & $41 \cdot 3^{b}$ & $8 \cdot 6^{b}$ & $64 \cdot 3$ & $16 \cdot 0$ & $19 \cdot 7^{\mathrm{a}}$ \\
\hline High-fibre diet (fine wholemeal rye bread) & $42 \cdot 7^{b}$ & $9 \cdot 1^{\mathrm{b}}$ & $65 \cdot 3$ & $19 \cdot 4$ & $15 \cdot 6^{\mathrm{ab}}$ \\
\hline $\mathrm{SE}$ & 1.3 & 0.3 & $2 \cdot 0$ & $2 \cdot 2$ & 1.5 \\
\hline \multicolumn{6}{|l|}{ Breads } \\
\hline Wheat-rye mixed bread & $44 \cdot 7$ & $10 \cdot 6^{\mathrm{a}}$ & $65 \cdot 8$ & $21.9^{a}$ & $12 \cdot 4^{b}$ \\
\hline Wholemeal rye bread (coarse) & $40 \cdot 3$ & $8 \cdot 8^{b}$ & 63.2 & $15 \cdot 7^{b}$ & $20 \cdot 9^{a}$ \\
\hline Wholemeal rye bread (fine) & 41.0 & $8 \cdot 4^{\mathrm{b}}$ & $64 \cdot 1$ & $20 \cdot 2^{a}$ & $15 \cdot 7^{\mathrm{b}}$ \\
\hline SE & $1 \cdot 3$ & 0.4 & $2 \cdot 3$ & $2 \cdot 3$ & 1.6 \\
\hline \multicolumn{6}{|l|}{ Difference between diet and corresponding bread } \\
\hline Low-fibre diet-wheat rye mixed bread & $2 \cdot 0$ & & $3.4^{*}$ & $-2 \cdot 4^{*}$ & $-1 \cdot 0$ \\
\hline High-fibre diet (coarse wholemeal rye bread)-coarse wholemeal rye bread & 1.0 & & $1 \cdot 1$ & $0 \cdot 3$ & $-1 \cdot 2$ \\
\hline High-fibre diet (fine wholemeal rye bread)-fine wholemeal rye bread & 1.7 & & $1 \cdot 1$ & $-0.9^{*}$ & $-0 \cdot 1$ \\
\hline SE & 0.9 & & 0.5 & 0.3 & 0.5 \\
\hline
\end{tabular}

a,b For each study and group of substrates, values within a column with unlike superscript letters were significantly different $(P<0.05)$. For details of statistical procedures see p. 33.

Values were significantly different from zero: ${ }^{*} P<0.05$. For details of statistical procedures see p. 33.

†For details of diets see p. 32 and Wisker et al. (1998).

$\ddagger$ Total SCFA, sum of acetate, propionate and butyrate.

$\S$ Polysaccharides, sum of non-starch polysaccharides and resistant starch.

bread $(P<0 \cdot 05)$ and the wheat-rye mixed bread $(P<0 \cdot 01)$. Although the butyrate ratio for the fine wholemeal bread was higher than that for the wheat-rye mixed bread, the difference was not significant $(P=0 \cdot 0880)$. The diet containing the coarse bread and the coarse bread itself were the only substrates leading to higher butyrate than propionate ratios. Significant differences between mixed diets and corresponding breads were observed for the ratio of acetate $(P<0.01)$ and propionate $(P<0.01)$ for the couple low-fibre diet/wheat-rye mixed bread and for the ratio of propionate for the couple high-fibre diet containing fine wholemeal rye bread/fine wholemeal rye bread $(P<0 \cdot 01)$.
Faecal SCFA during the ingestion of the experimental diets and the differences between in vitro and faecal SCFA molar ratios are shown in Table 3. Although the concentrations of faecal SCFA differed between the single diets, the differences were not significant because of large individual variations. Molar ratios of butyrate were enhanced during the intake of the diets containing wholemeal bread, but the differences to the diet containing wheat-rye mixed bread were not significant (coarse bread diet $P=0.0903$, fine bread diet $P=0.3294$ ). Similarly to in vitro, the high-fibre diet containing coarse bread led to higher faecal butyrate than propionate ratios. For all three diets, there were only

Table 3. Concentrations and molar ratios of faecal short-chain fatty acids (SCFA) and differences between in vitro and faecal molar ratios of SCFA* $\dagger$

(Least squares means with their standard errors for seven subjects)

\begin{tabular}{|c|c|c|c|c|}
\hline & \multirow{2}{*}{$\begin{array}{c}\text { Total SCFA } \ddagger \\
\text { ( } \mu \mathrm{mol} / \mathrm{g} \text { wet faeces) }\end{array}$} & Acetate & Propionate & Butyrate \\
\hline & & \multicolumn{3}{|c|}{ Molar ratio } \\
\hline Low-fibre diet & $64 \cdot 7$ & 63.5 & $21 \cdot 6$ & 14.9 \\
\hline High-fibre diet (coarse wholemeal bread) & $75 \cdot 6$ & $62 \cdot 3$ & 17.6 & $19 \cdot 6$ \\
\hline High-fibre diet (fine wholemeal bread) & $95 \cdot 8$ & 61.5 & $20 \cdot 4$ & $17 \cdot 7$ \\
\hline SE & $15 \cdot 7$ & $3 \cdot 1$ & $2 \cdot 1$ & $1 \cdot 7$ \\
\hline \multicolumn{5}{|c|}{ Difference between in vitro and faecal molar ratios of SCFA } \\
\hline Low-fibre diet & & $5 \cdot 6$ & $-2 \cdot 0$ & -3.5 \\
\hline High-fibre diet (coarse wholemeal bread) & & $2 \cdot 0$ & $-1 \cdot 7$ & 0.1 \\
\hline High-fibre diet (fine wholemeal bread & & 3.9 & $-1 \cdot 1$ & $-2 \cdot 0$ \\
\hline SE & & 1.9 & 1.5 & $1 \cdot 8$ \\
\hline
\end{tabular}

* Differences between concentrations and molar ratios of SCFA in faeces were not significant. For details of the statistical procedure see p. 33 . †Differences between faecal and in vitro molar ratios of SCFA were not significantly different from zero. For details of statistical procedures see p. 33.

‡SCFA, sum of acetate, propionate, and butyrate. 
non-significant differences between faecal and in vitro molar ratios of acetate, propionate and butyrate; the overall agreement was best for the high-fibre diet containing coarse wholemeal bread.

\section{Discussion}

After $24 \mathrm{~h}$ in vitro fermentation of substrates obtained from mixed diets the amounts and patterns of SCFA varied depending on the types of bread present in the diets. Bread contributed more than $80 \%$ to total NSP in the high-fibre diets. Accordingly, there were only small differences in the SCFA patterns between these diets and the corresponding breads. For the low-fibre diet that contained a lower proportion of bread NSP $(60 \%)$, there were greater differences between diet and bread SCFA patterns.

In vitro fermentations were performed for 24 and $48 \mathrm{~h}$ (Wisker et al. 1998), but we evaluated the results with respect to SCFA production only for the $24 \mathrm{~h}$ incubations. After that time the extent of NSP breakdown in vitro agreed rather well with the degradation measured in vivo, whilst after $48 \mathrm{~h}$ the breakdown of NSP in vivo was significantly overestimated (Wisker et al. 1998). Furthermore, in a static in vitro system after long incubation times substrates become depleted and bacterial metabolites accumulate, some of which may inhibit the bacterial acitivity (Brøbech Mortensen et al. 1991; Macfarlane et al. 1994).

It cannot be excluded that the dilution of the freshly voided faeces with water (1:5) and the concentration of inocula $(40 \mathrm{~g} / \mathrm{l}) \mathrm{had}$ an influence on the activity of the faecal bacteria in vitro. However, previous in vitro studies showed that SCFA formation was not altered with osmolarity changes in the range of 50 (dilution of faeces with plain water) to $600 \mathrm{mosmol}$, but was completely inhibited above 2000 mosmol/1 (Brøbech Mortensen et al. 1991). Inoculum concentrations used in other studies varied. Adiotomre et al. (1991) and Weaver et al. (1992), for example, used the same or similar inoculum concentrations (40 and $50 \mathrm{~g} / \mathrm{l}$ respectively) as we did, but in other studies fermentations were performed with inoculum concentrations between 10 (McBurney \& Thompson, 1989) and 250 g/l (Salvador et al. 1993). The good agreement between the extent of in vivo and in vitro NSP degradation after $24 \mathrm{~h}$ incubation (Wisker et al. 1998) suggests that the inocula had sufficient activity.

On a weight for weight basis, approximately $60-70 \%$ of hexose monosaccharides can theoretically be converted to SCFA (Miller \& Wolin, 1979; Cummings, 1994, 1995). Taking the molar ratios of SCFA into account, for the lowfibre diet and the diets containing coarse or fine wholemeal rye bread, $0.62,0.52$ and $0.53 \mathrm{~g}$ SCFA respectively, were produced per $\mathrm{g}$ fermented monosaccharide. The corresponding values for the wheat-rye mixed bread, the coarse and fine wholemeal rye bread were $0.63,0.54$ and $0.51 \mathrm{~g}$ respectively. Thus, for the high-fibre diets and the corresponding breads the SCFA yields were lower than the theoretically possible amounts, while the conversion was more efficient for the substrates prepared from the low-fibre diet and the wheat-rye mixed bread. The reasons for these differences are not obvious; probably they are related to differences in the types of polysaccharides fermented.
The SCFA patterns obtained in vitro were influenced by the particle size of the breads, although the particle size had no effect on the extent of NSP degradation (Wisker et al. 1998). The percentages of butyrate measured for the coarse wholemeal bread-containing diet and the coarse wholemeal bread alone were similar to values reported for wheat and oat bran (McBurney \& Thompson, 1990; Adiotomre et al. 1991; Weaver et al. 1992). The butyrate ratios for the fine wholemeal bread-containing diet and the fine bread, however, were lower. With the coarse wholemeal bread-containing diet and the coarse bread about twice the amounts of RS were fermented compared with the fine whole bread-containing diet and the fine bread. Starch presumably included in coarse particles escaped the enzymic hydrolysis during the preparation of the residues, and hence acted as fermentation substrate. Starch was shown to yield relatively high molar proportions of butyrate (mean $22 \%$ ) (Cummings, 1995).

Although the amounts of fermented RS were similar for the low-fibre wheat-rye mixed bread and the fine wholemeal rye bread, the latter yielded a higher ratio of butyrate (although only borderline significant, $P=0.088$ ). This in vitro finding may support results obtained in rats, in which replacing white bread by increasing amounts of wholemeal bread led to increasing production of butyrate in the caecum (Key \& Mathers, 1993). Compared with the wheat-rye mixed bread, for the substrates prepared from wholemeal rye bread more pentoses (arabinoxylans) were fermented, which in part originated from the outer layers of the rye grain (Selvendran, 1983). Recent studies in pigs on different milling fractions of rye indicate that comparatively high butyrate ratios may be ascribed to NSP present in the aleurone layer (Glits $\varnothing$ et al. 1998).

The concentrations of faecal SCFA during the consumption of the experimental diets were in the range of those reported by other authors (Cummings et al. 1996; Hylla et al. 1998), but as in many previous studies (e.g. Cummings et al. 1996; Heijnen et al. 1998; Hylla et al. 1998) they were not significantly affected by the diet consumed, most likely because most of the SCFA produced are rapidly absorbed (Ruppin et al. 1980). The molar proportions of faecal SCFA, however, can be influenced by the substrates available in the distal gut (McIntyre et al. 1991; Macfarlane et al. 1994). The higher butyrate ratios (although not significantly) for the wholemeal bread-containing diets compared with those for the wheat-rye mixed bread-containing diet probably indicate that parts of the NSP in the wholemeal breads resisted fermentation in the proximal colon and served as substrates for the bacteria in the distal gut. This would agree with results in rats in which fermentation of wheat bran persisted until the distal large bowel (McIntyre et al. 1991). Faecal starch excretion was low and not significantly different between the diets, although there was a tendency to higher losses for the coarse bread diet (Wisker et al. 1996). So it is not clear whether the somewhat higher ratio of faecal butyrate for the coarse compared with the fine wholemeal bread diet was due to more starch reaching the distal colon with coarse particles.

For a given substrate, the molar ratios of SCFA did not change markedly between 12 and $24 \mathrm{~h}$ of in vitro fermentation (McBurney \& Thompson, 1990). If this were also the 
case in vivo, the best agreement between in vitro and faecal SCFA pattern might be expected for polysaccharides the fermentation of which could persist to the distal end of the bowel. For the diets investigated, there were no significant differences between in vitro and corresponding faecal SCFA patterns, but the agreement was best for the diet containing the coarse wholemeal bread. The NSP in this latter diet probably was more slowly fermented than NSP in the other diets, because the coarse particles might have impeded the access to bacterial enzymes.

In conclusion, our studies showed that after $24 \mathrm{~h}$ in vitro fermentation the SCFA patterns obtained for total diet residues were influenced by the breads present in the diets. The degree of extraction, i.e. bread prepared from endosperm $v$. wholemeal flour, and the particle size had an effect on the molar ratios of SCFA produced in vitro. The type of bread had also an effect on faecal SCFA pattern, but the differences between the different breadcontaining diets were less pronounced than in vitro. For the diets investigated, in vitro and faecal SCFA pattern were similar.

\section{Acknowledgements}

Special appreciation goes to the subjects for their excellent cooperation. We thank K. Golz and A. Richardsen for preparing the diets and W. Seibel and J. Brümmer, Federal Institute of Grain, Potato and Fat Research, Detmold, Germany, for the supply of the wholemeal rye breads. Part of the food was kindly provided by Union Deutsche Lebensmittelwerke, Hamburg, Germany. We are grateful to A. Susenbeth and S. Wolffram, Institute of Animal Nutrition, University of Kiel, for the opportunity to perform parts of the chemical analyses in their Institute.

\section{References}

Adiotomre J, Eastwood MA, Edwards CA \& Brydon WG (1990) Dietary fiber: in vitro methods that anticipate nutrition and metabolic activity in humans. American Journal of Clinical Nutrition 52, 128-134.

Bingham SA (1996) Epidemiology and mechanisms relating diet to risk of colorectal cancer. Nutrition Research Reviews 9, 197 239.

Brøbech Mortensen P, Hove H, Rye Clausen M \& Holtug K (1991) Fermentation to short-chain fatty acids and lactate in human faecal batch cultures. Intra- and inter- individual variations versus variations caused by changes in fermented saccharides. Scandinavian Journal of Gastroenterology 26, 1285-1294.

Cummings JH (1994) Quantitating short chain fatty acid production in humans. In Short Chain Fatty Acids, pp. 11-19 [HJ Binder, J Cummings and KH Soergel, editors]. Dordrecht, The Netherlands: Kluwer Academic Publishers.

Cummings JH (1995) Short-chain fatty acids. In Human Colonic Bacteria: Role in Nutrition, Physiology, and Pathology, pp. 101-130 [GR Gibson and GT Macfarlane, editors]. Boca Raton, FL: CRC Press.

Cummings JH, Beatty ER, Kingman SM, Bingham SA \& Englyst HN (1996) Digestion and physiological properties of resistant starch in the human large bowel. British Journal of Nutrition $\mathbf{7 5}$, 733-747.

Englyst HN, Hay S, Macfarlane GT (1987) Polysaccharide breakdown by mixed populations of human faecal bacteria. FEMS Microbiology and Ecology 95, 163-171.

Glits $\varnothing$ LV, Brunsgaard G, Højsgaard S, Sandstöm B \& Bach Knudsen KE (1998) Intestinal degradation in pigs of rye dietary fibre with different structural characteristics. British Journal of Nutrition 80, 457-468.

Goering HK \& Van Soest PJ (1970) Forage Fiber Analyses. Agriculture Handbook no. 379. Washington, DC: United States Department of Agriculture, U.S. Government Printing Office.

Heijnen ML-A, van Amelsvoort, JMM, Deurenberg P \& Beynen AC (1998) Limited effect of consumption of uncooked $\left(\mathrm{RS}_{2}\right)$ or retrograded $\left(\mathrm{RS}_{3}\right)$ resistant starch on putative risk factors for colon cancer in healthy men. American Journal of Clinical Nutrition 67, 322-331.

Hylla S, Gostner A, Dusel G, Anger H, Bartram H-P, Christl SU, Kasper H \& Scheppach W (1998) Effect of resistant starch on the colon in healthy volunteers: possible implications for cancer prevention. American Journal of Clinical Nutrition 67, 136142.

Key FB \& Mathers JC (1993) Gastrointestinal responses of rats fed on white and wholemeal breads: complex carbohydrate digestibility and the influence of dietary fat content. British Journal of Nutrition 69, 481-495.

McBurney MI \& Thompson LU (1989) Effect of human faecal donor on in vitro fermentation variables. Scandinavian Journal of Gastroenterology 24, 359-367.

McBurney MI \& Thompson LU (1990) Fermentative characteristics of cereal brans and vegetable fibers. Nutrition and Cancer 13, 271-280.

Macfarlane GT, Gibson GR \& Macfarlane S (1994) Short chain fatty acid and lactate production by human intestinal bacteria grown in batch and continuous culture. In Short Chain Fatty Acids, pp. 44-60 [HJ Binder, J Cummings and KH Soergel, editors]. Dordrecht, The Netherlands: Kluwer Academic Publishers.

McIntyre A, Young GP, Taranto T, Gibson PR \& Ward PB (1991) Different fibers have different regional effects on luminal contents of rat colon. Gastroenterology 101, 1274-1281.

Miller TL \& Wolin MJ (1979) Fermentations by saccharolytic intestinal bacteria. American Journal of Clinical Nutrition 32, 164-172.

Prosky L, Asp N-G, Furda I, DeVries JH, Schweizer TF \& Harland B (1985) Determination of total dietary fiber in foods and food products: collaborative study. Journal of the Association of Official Analytical Chemists 68, 677-679.

Roediger WEW (1982) Utilization of nutrients by isolated epithelial cells of the rat colon. Gastroenterology 83, 424-429.

Ruppin H, Bar-Meir S, Soergel KH, Wood CM \& Schmitt MG Jr (1980) Absorption of short-chain fatty acids by the colon. Gastroenterology 78, 1500-1507.

Salvador V, Cherbut C, Barry JL, Bertrand D, Bonnet C \& Delort-Laval J (1993) Sugar composition of dietary fibre and short-chain fatty acid production during in vitro fermentation by human bacteria. British Journal of Nutrition 70, 189197.

Scheppach W, Fabian M, Sach M \& Kasper HJ (1988) The effect of starch malabsorption on fecal short-chain fatty acid excretion in man. Scandinavian Journal of Gastroenterology 23, 755759.

Selvendran RR (1983) The chemistry of plant cell walls. In Dietary Fiber, pp. 95-147 [GG Birch and KJ Parker, editors]. New York, NY: Applied Science Publishers.

Van Soest PJ, Jeraci J, Foose T, Wrick K \& Ehle F (1982) Comparative fermentation of fibre in man and other animals. In Fibre in Human and Animal Nutrition, pp. 75-80 [G Wallace and L Bell, editors]. Palmerston North, New Zealand: The Royal Society of New Zealand. 
Weaver GA, Krause JA, Miller TL, Wolin MJ (1992) Cornstarch fermentation by the colonic microbial community yields more butyrate than does cabbage fiber fermentation; cornstarch fermentation rates correlate negatively with methanogenesis. American Journal of Clinical Nutrition 55, 70-77.

Wisker E, Daniel M \& Feldheim W (1996) Particle size of whole meal rye bread does not affect the digestibility of macro-nutrients and non-starch polysaccharides and the energy value of dietary fibre in humans. Journal of the Science of Food and Agriculture 70, 327-333.

Wisker E, Daniel M, Rave G \& Feldheim W (1998) Fermentation of non-starch polysaccharides in mixed diets and single fibre sources: comparative studies in human subjects and in vitro. British Journal of Nutrition 80, 253-261. 